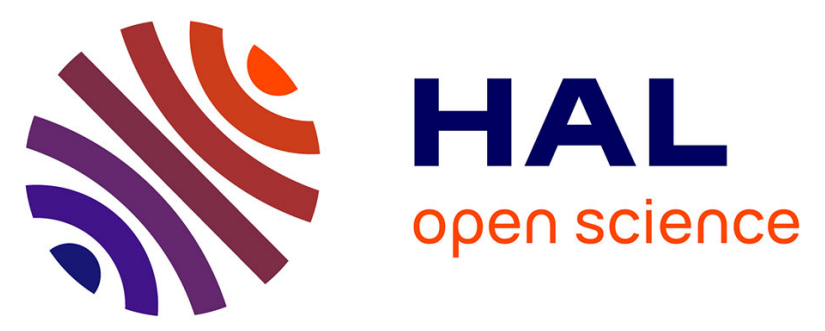

\title{
A biophysical model of intracellular distribution and perinuclear accumulation of particulate matter
}

Ilaria Rivolta, Alice Panariti, Maddalena Collini, Barbara Lettiero, Laura

d'Alfonso, Laura Sironi, Giuseppe Miserocchi, Giuseppe Chirico

\section{- To cite this version:}

Ilaria Rivolta, Alice Panariti, Maddalena Collini, Barbara Lettiero, Laura d'Alfonso, et al.. A biophysical model of intracellular distribution and perinuclear accumulation of particulate matter. Biophysical Chemistry, 2011, 158 (2-3), pp.134. 10.1016/j.bpc.2011.06.009 . hal-00778541

\section{HAL Id: hal-00778541 \\ https://hal.science/hal-00778541}

Submitted on 21 Jan 2013

HAL is a multi-disciplinary open access archive for the deposit and dissemination of scientific research documents, whether they are published or not. The documents may come from teaching and research institutions in France or abroad, or from public or private research centers.
L'archive ouverte pluridisciplinaire HAL, est destinée au dépôt et à la diffusion de documents scientifiques de niveau recherche, publiés ou non, émanant des établissements d'enseignement et de recherche français ou étrangers, des laboratoires publics ou privés. 


\section{Accepted Manuscript}

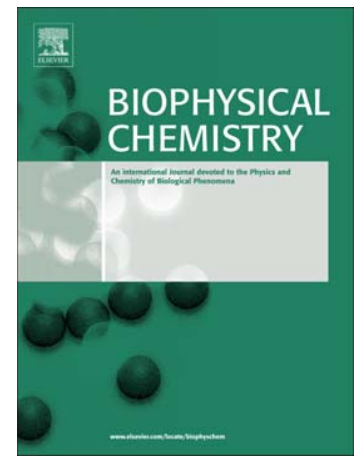

A biophysical model of intracellular distribution and perinuclear accumulation of particulate matter

Ilaria Rivolta, Alice Panariti, Maddalena Collini, Barbara Lettiero, Laura D'Alfonso, Laura Sironi, Giuseppe Miserocchi, Giuseppe Chirico

PII: $\quad$ S0301-4622(11)00206-7

DOI: $\quad$ doi: $10.1016 /$ j.bpc.2011.06.009

Reference: $\quad$ BIOCHE 5540

To appear in: Biophysical Chemistry

Received date: 2 June 2011

Revised date: $\quad 16$ June 2011

Accepted date: $\quad 16$ June 2011

Please cite this article as: Ilaria Rivolta, Alice Panariti, Maddalena Collini, Barbara Lettiero, Laura D'Alfonso, Laura Sironi, Giuseppe Miserocchi, Giuseppe Chirico, A biophysical model of intracellular distribution and perinuclear accumulation of particulate matter, Biophysical Chemistry (2011), doi: 10.1016/j.bpc.2011.06.009

This is a PDF file of an unedited manuscript that has been accepted for publication. As a service to our customers we are providing this early version of the manuscript. The manuscript will undergo copyediting, typesetting, and review of the resulting proof before it is published in its final form. Please note that during the production process errors may be discovered which could affect the content, and all legal disclaimers that apply to the journal pertain. 


\section{A biophysical model of intracellular distribution and perinuclear accumulation of particulate matter}

Ilaria Rivolta ${ }^{l}$, Alice Panariti ${ }^{1}$, Maddalena Collini ${ }^{2}$, Barbara Lettiero ${ }^{1}$, Laura D'Alfonso ${ }^{2}$, Laura Sironi ${ }^{2}$, Giuseppe Miserocchi ${ }^{1}$, Giuseppe Chirico ${ }^{2}$

${ }^{1}$ Dipartimento di Medicina Sperimentale, Università di Milano Bicocca, Via Cadore, 48 - 20052 Monza (I)

${ }^{2}$ Dipartimento di Fisica, Università di Milano Bicocca, Piazza della Scienza 3, 20126 Milano (I).

Corresponding Author: Giuseppe Chirico

Dipartimento di Fisica, Piazza della Scienza 3, 20126, Milano

giuseppe.chirico@mib.infn.it; tel: +39-0264482440; Fax: +39-0264482585

http://fisica.mib.infn.it/pages/home.php 


\section{Abstract}

We have measured in human alveolar cells the cytoplasmic distribution of the fluorophore coumarin-6 carried by Solid Lipid Nanoparticles (SLNs) and observed a perinuclear accumulation of the fluorescence that can be described by a single exponential growth along an ideal line joining the plasma membrane to the nuclear border and by a sigmoidal relationship as a function of time. Intracellular distribution was affected by lowering the temperature from 37 to $4^{\circ} \mathrm{C}$ and by the disruption of cytoskeleton by cytochalasin $\mathrm{D}$, but it was minimally perturbed by the inhibition of ATP dependent molecular motors. A biophysical model was developed for an accumulation of loaded particles against a diffusion gradient based on a mean field interaction energy, whose origin we ascribe to the actin structure of the cytoskeleton. The estimated value for the load diffusion coefficient was four and two orders of magnitude less than that of free coumarin- 6 and of SLNs in aqueous solutions, respectively, suggesting that the load moves within the cell cytoplasm in a form still reminiscent of the nanocarrier structure. 


\section{Introduction}

The uptake of extracellular material across the plasma membrane of eukaryotic cells is well described and characterized by a variety of mechanisms going from the passive diffusion to the active transport [1]. The former, defined as a concentration gradient-driven mass transport of a compound, is considered the dominant route [1]. The latter, including the endocytosis mediated processes, is in charge of taking up larger molecules and molecular complexes.

Once entered the cells, the intracellular traffic and distribution of substances that do not follow the endocytotic pathway are related to aspecific movements or microtubule-based active mechanisms [2,3]. Furthermore, exogenous material of various origin tends to accumulate in the perinuclear region after cellular uptake [2,4-9]. In a recent study involving human alveolar epithelial cell line, we confirmed this observation also for Solid Lipid Nanoparticles, SLNs, loaded with the fluorophore coumarin-6 (c-SLNs) and we hypothesized a pathway for their uptake [10].

Here we want to analyse, from a biophysical point of view, the cytoplasmic distribution of the fluorophore carried by the SLNs, and the way it is affected by the incubation temperature, by the state of the cytoskeleton and the efficiency of the molecular motors. We focused our efforts on the perinuclear accumulation of coumarin-6, taken here as a model hydrophobic compound and develop a biophysical model of the fluorophore distribution in the cell that assumes an interaction energy between the fluorophore carried by the SLNs and cellular components. This interaction energy counterbalances the free thermal diffusion of the molecules within the cytoplasm and could be specifically related to the cytoskeleton or to active transport mechanisms such as those related to the molecular motors. The modelling of the perinuclear accumulation of nanoparticulate that we outline here, may be of potential interest for the biomedical use of nanoparticulate structures, in particular for the delivery of drugs or genetic material in the nuclear region.

\section{Materials and methods}

Nanoparticles. SLNs were produced by NANOVECTOR, by choosing tripalmitin as lipidic matrix. SLNs have been loaded with coumarin-6 (c-SLNs) to allow their visualization by means of fluorescence microscopy $\left(\lambda_{\mathrm{exc}}=450 \mathrm{~nm}, \lambda_{\mathrm{em}}=505 \mathrm{~nm}\right)$. The molar ratio coumarin-6:tripalmitin was about 80 (NANOVECTOR, personal communication). The average diameter of heat sterilised c-SLNs dispersion, 
determined by Dynamic Light Scattering (Brookhaven 90Plus system, Brookhaven Instruments Corp.), was $116 \pm 15 \mathrm{~nm}$, and remained essentially stable $(\leq 130 \mathrm{~nm})$ either when the particles were dispersed in buffer or in cell culture medium supplemented with $1 \%$ FBS. The $\mathrm{Z}$ potential of the SLNs was $-24 \pm 2 \mathrm{mV}$ (Brookhaven 90Plus system, Brookhaven Instruments Corp).

In experiments where coumarin-6 was used as free drug, a stock solution of $20 \mu \mathrm{g} / \mathrm{ml}$ in DMSO was prepared and diluted at the desired working concentration.

Cell culture. A30 cells [11] were grown on Petri dishes in DMEM medium supplied with $10 \%$ FBS, $1 \%$ of L-Glutamine and $1 \%$ of Penicillin/Streptomycin and incubated in a controlled environment at $37^{\circ} \mathrm{C}$ with $5 \%$ $\mathrm{CO}_{2}$. During the experiments the cells were incubated with medium supplemented with $1 \%$ FBS to prevent NPs aggregation.

Effect of temperature on NPs intracellular distribution. We estimated the cytoplasmic concentration of coumarin- 6 by monitoring the fluorescence distribution in cells exposed to c-SLNs $(0.01 \mathrm{mg} / \mathrm{ml})$ for $45 \mathrm{~min}$ either at $37^{\circ} \mathrm{C}$ or at $4^{\circ} \mathrm{C}$. In the latter case the cells were pre-cooled for $30 \mathrm{~min}$ at $4^{\circ} \mathrm{C}$. At the end of the incubation period, cells were immediately fixed in paraformaldehyde $4 \%$ in PBS.

Image acquisition. The experimental set up consisted of a wide field NIKON Eclipse FN1 equipped with a 63X objective, numerical aperture 1.2, motorized table (Prior Scientific, Rockland, MA, USA), Photometric coolSNAP ES Digital Camera and Metamorph (Molecular Device, Downington, PA, USA) software for image acquisition and analysis. We used fields of view that encompassed at least 5 cells for analysis. The fluorescence signal form the c-SLNs was discriminated by a band pass filter $\left(\lambda_{\mathrm{em}}=505 \mathrm{~nm}\right)$ and the images were averaged over an exposure time of 400 milliseconds.

Starting from the top of the cell, $40 \mathrm{Z}$ planes were acquired along the $\mathrm{Z}$ axis at $0.5 \mu \mathrm{m}$ inter-distance. We typically considered the overall fluorescence acquired in the wide field. In specific cases, we used the fluorescence estimated on a $\mathrm{Z}$ plane corresponding to the mid cell height, obtained through a deconvolution process (AutoDeblur, Media Cybernetics, Bethesda, MA, USA).

Drugs treatment: The role of cytoskeleton in affecting fluorescence cytoplasmic distribution was addressed by treating cells, previously exposed to c-SLNs $\left(0.01 \mathrm{mg} / \mathrm{ml}\right.$ for 45 minute at $\left.37^{\circ} \mathrm{C}\right)$ and analysing the perinuclear fluorescence that these cells displayed when incubated with cytochalasin $D(C y t o D)$ at a concentration of $2 \mu \mathrm{M}$ for 1 hour at $37^{\circ} \mathrm{C}$. In other experiments, before the exposure to the NPs, cellular 
energy stores were depleted by a pre-treatment for $1 \mathrm{~h}$ at $37^{\circ} \mathrm{C}$ with $0.2 \mathrm{mM}$ sodium azide $\left(\mathrm{NaN}_{3}\right)$ or $50 \mathrm{mM}$ 2-deoxy-D-glucose (DDG) [12]. After this pre-treatment, the cells were incubated also with c-SLN (0.01 $\mathrm{mg} / \mathrm{ml}$ ) for additional 45 minute at $37^{\circ} \mathrm{C}$. At the end of the incubation time the intracellular fluorescence distribution was monitored and the images were acquired and analysed as described. Nuclei were labelled with DAPI $1 \mu \mathrm{M}$.

Immunocytochemistry. Cells plated on glass coverslips were fixed with paraformaldehyde $4 \%$ in PBS at room temperature for 20 min, washed three times in PBS, LS (Low Salt PBS) and in HS (High Salt PBS) respectively and then permeabilized with digitonin $0.01 \%$ in GDB for $30 \mathrm{~min}$. Fixed and permeabilized cells were incubated with phalloidin conjugated with Texas RED (dil 1:200. Molecular Probes) for actin filaments staining or primary antibody antimyosin (dil 1:500, Covance) diluted in GDB at RT for 2 hours. After washing with HS for three times, cells labeled for myosin were incubated with Alexa conjugated secondary antibody diluted in GDB (1:100). Cells were then washed with HS AND LS three times respectively. DAPI was used to stain cell nuclei at a concentration of $1 \mu \mathrm{M}$ in PBS for $5 \mathrm{~min}$. Coverslips were mounted with glycerol.

Statistical analysis: Statistical analysis were carried out by t-test and the significance level was set at $\mathrm{p}<0.001$ (indicated by symbol * or \# in the figures).

\section{Results}

The intracellular fluorescence distribution of the fluorophores loaded in the c-SLNs was measured on monolayers of human lung epithelial A30 cells, very similar to the more studied A549,[11] an important component of the blood-air barrier and a sensitive site for particulate-body interaction. The analysis of the coumarin-6 fluorescence distribution was performed by selecting, on individual cells, a line (emission line, EL) running from the plasma to the nuclear membrane (Fig 1, image) and four cytoplasmic regions of interest (10 pixel square ROIs) positioned along such EL at $25 \%, 50 \%$ and $75 \%$ of the total length. The location of the plasma membrane was obtained by saturating the colour levels to half the maximum colour depth in the images. We verified that this allowed us to locate the cell membrane by comparing with images in which the actin filaments were specifically labelled. Since the width of the cell cytoplasm varies among the cells we needed to define a normalized distance along the EL in order to compare and average the 
fluorophore distributions measured on different cells. Therefore in the following we will refer to the distance along the EL as $x=100\left(r-r_{N}\right) /\left(r_{C}-r_{N}\right)$, where $r_{N}, r_{C}$ and $r$ are the positions of the nuclear and the cell membrane and the selected ROI along the EL ( $x=100 \%$ indicates the nuclear membrane).

The cells were exposed to an estimated concentration of $1.8 \times 10^{10} \mathrm{c}-\mathrm{SLNs} / \mathrm{ml}$, corresponding to a tripalmitin concentration $\cong 0.01 \mathrm{mg} / \mathrm{ml}$, one order of magnitude lower than the toxic concentration $(0.2$ $\mathrm{mg} / \mathrm{ml}$ ) estimated by LDH release assay and MTT test.[10]

\section{Cytoplasmic distribution of c-SLNs.}

We followed the kinetics of c-SLNs intracellular distribution by monitoring the ROI's average fluorescence signal along the EL line at 3, 9, 15 and 30 minutes after the addition of NPs to the cell culture $\left(\mathrm{T}=32^{\circ} \mathrm{C}\right.$ during the images acquisition, Fig.2). A minimum of seven cells was used to average the data. The intracellular fluorescence increased as a function of the distance from the plasma membrane, reaching the maximum value in the proximity of the nuclear membrane (Fig.2A). The fluorescence spatial distribution, $\mathrm{F}(\mathrm{x})$, observed during the uptake process and at equilibrium (Fig.2A), can be described by an exponential growth as a function of the distance, $\mathrm{x}$, from the cell membrane along the EL according to:

$$
F(x)=F_{0}+A \exp [(x-100) / L]
$$

It is important to notice that the cell membrane value of the fluorescence is $F(0) \cong F_{0}+A \exp [-100 / L]$. The best fit parameters of the solid lines reported in Fig.2A are: A $(15 \mathrm{~min})=135 \pm 40$ [arbitrary units, a.u.], $\mathrm{L}(15 \mathrm{~min})=26 \pm 5 \%$ and $\mathrm{F}_{0}(15 \mathrm{~min})=12 \pm 5 \quad$ [a.u.], and $\mathrm{A}(30 \mathrm{~min})=330 \pm 50 \quad$ [a.u.], $\mathrm{L}(30 \mathrm{~min})=29 \pm 3 \%$ and $\mathrm{F}_{0}(30 \mathrm{~min})=10 \pm 2$ [a.u.].

The only relevant difference in the time evolution of the fluorescence distribution concerns the inhomogeneity ratio $\mathrm{A} / \mathrm{F}_{0}$, that offers a quantification of the perinuclear accumulation of the fluorophore. This ratio varies from $12 \pm 7$ to $25 \pm 7$ when passing from $15 \mathrm{~min}$ to $30 \mathrm{~min}$ of c-SLNs exposure time, indicating a progressive perinuclear accumulation. The fluorescence signal followed in time at $\mathrm{x}=50 \%$ and $75 \%$ along the EL (Fig.2B) increases at first gradually and seems to reach a saturation value that depends on the position along the EL after approximately $30 \mathrm{~min}$ (Fig.2B). Such bimodal behaviour, can be fit to the following sigmoidal function: 


$$
f(t)=f_{0}+\frac{\left(f_{1}-f_{0}\right)}{1+\exp \left(\left(t-t_{1 / 2}\right) / \delta\right)}
$$

The mid-point time, $t_{1 / 2}$, is relatively insensitive to the position along the EL line, $t_{1 / 2} \cong 18 \mathrm{~min}(\mathbf{F i g} .2 \mathbf{B})$. The transition is instead sharper $(\delta(50 \%) \cong 3.5 \mathrm{~min}$ compared to $\delta(75 \%) \cong 7 \mathrm{~min})$ as we move closer to the cell membrane through which the c-SLNs carried fluorophore is internalized. This observation is an indication that the kinetics of the fluorophore distribution in the cytoplasm is affected also by the internalization process, as discussed later.

\section{Effect of temperature}

In order to evaluate the effect of the temperature on the distribution of the fluorophore, the cells were exposed to c-SLNs for 45 minutes either at $4^{\circ} \mathrm{C}$ or $37^{\circ} \mathrm{C}$, fixed as described in the Materials and Methods, and the distribution of the fluorophore was then measured (Fig. 3). The absolute value of the intracellular fluorescence is lower for cells incubated with the c-SLNs at the lowest temperature: we find $\mathrm{F}(75 \%)=155 \pm 10$ [a.u.] at $\mathrm{T}=37^{\circ} \mathrm{C}$ and $\mathrm{F}(75 \%)=80 \pm 12$ [a.u.] at $\mathrm{T}=4^{\circ} \mathrm{C}$. Changes in the cell thickness due to temperature do not affect substantially this conclusion since the emission $\mathrm{F}(75 \%)$ computed on the deconvoluted images (focal plane at $50 \%$ of the cell thickness) confirms a marked difference between the two temperatures investigated (350 \pm 80 [a.u.] compared to $262 \pm 6$ [a.u.] for $37^{\circ} \mathrm{C}$ and $4^{\circ} \mathrm{C}$ respectively, $\mathrm{p}<0.001,22$ cells). Moreover, the total amount of fluorophore loaded by the cell at $\mathrm{T}=37^{\circ} \mathrm{C}$, as measured by the integral under the lines reported in Fig.3, is approximately twice (8800 [a.u.]) that measured for $\mathrm{T}=4^{\circ} \mathrm{C}$ (4800 [a.u.]).

The intensity normalized to its maximum value increases with the distance from the cell membrane independent of the experimental temperature, and it can still be described by Eq. 1. The best fit decay length values are $\mathrm{L}=31 \pm 1 \%$ and $\mathrm{L}=43 \pm 2 \%$ for $\mathrm{T}=37^{\circ} \mathrm{C}$ and $\mathrm{T}=4^{\circ} \mathrm{C}$, respectively.

$\underline{\text { Role of molecular motors in determining the intracellular fluorescence distribution. }}$

The reduction of the cytoplasmic fluorescence intensity observed at $4{ }^{\circ} \mathrm{C}$ might reflect the temperature dependent inhibition of active ATP-dependent mechanisms, the so called "molecular motors" that are known to promote cell intracellular transport [13]. In order to assess the role of the molecular 
motors, we depleted cells from ATP, applying $\mathrm{NaN}_{3}$ or DDG before and during the incubation with the cSLN. Despite the inhibition of energy production by the metabolic poisons, as Fig. 4 shows, we still found the characteristic intracellular distribution and perinuclear accumulation of the fluorophore. Role of cytoskeleton on the intracellular distribution

In order to probe the role of cytoskeleton on the steady state fluorescence distribution, we incubated the cells with cytochalasin $\mathrm{D}$ (cytoD) at a concentration $(2.0 \mu \mathrm{M}$ for 1 hour) [8] suitable to disintegrate actin bundles but low enough to avoid cytotoxic damage, as measured by a lactate dehydrogenase releasing assay (data not shown). We found that the treatment with cytoD led to a more homogeneous pattern of the fluorescence signal inside the cells, suggesting that the fluorophore undergoes a redistribution in the cytoplasm (Fig. 5A,A'). While in the physiological condition the perinuclear/cytoplasmic fluorescence ratio increased 5 fold, in the CytoD treated cells, this ratio reaches now only a plateu value $\cong 1.7$ (Fig.5E). A similar ratio, $\cong 1.5$, was obtained when CytoD was applied before and maintained during the incubation of cells with c-SLN (Fig.5E). It is worth noting that, upon cytoD treatment, not only the actin filaments (Fig.5B,B') but also the myosin fibres were distrupted in the cells (Fig.5C,C'), while the microtubules remained almost intact (Fig.5D,D').

\section{Discussion}

Our data indicate that when A30 epithelial cells are exposed to c-SLNs, the intracellular fluorescence signal progressively increases (20-80 fold) along the EL line with a monotonic accumulation in the perinuclear region. As can be observed from Figs. 2 and $\mathbf{3}$, at least two processes are active in determining this inhomogeneous distribution, with a cross-over at $\mathrm{x} \cong 30 \%$ along the EL that depends on the incubation temperature (see Fig.3). The experiments with metabolic poisons $\left(\mathrm{NaN}_{3}\right.$ and DDG) do not substantially modify the cytoplasmic fluorescence distribution (Fig.4) In fact, as from the plot in Fig.4, the effect of $\mathrm{NaN}_{3}$ and DDG correspond to maximum decreases, with respect to the control cell line, of $14 \pm 18 \%$ and $22 \pm 26$ $\%$, respectively. Though these values indicate a slight systematic decrease of the perinuclear accumulation effect when treating the cells with metabolic poisons, this effect is still largely within the experimental uncertainties. The increase in the fluorescence signal when approaching the nuclear membrane is still relevant, being $\cong 7,6$ and 5 for the control line and the lines treated with $\mathrm{NaN}_{3}$ and DDG, respectively. 
Therefore, the data reported in Fig.4 do not allow us to assume a clear direct effect of the molecular motors on the perinuclear accumulation of the fluorophore at least in the present case in which SLNs have been used as extracellular carriers. However, it is worth noting that a minor direct role of the molecular motors in the generation of the perinuclear accumulation cannot be completely ruled out.[14]

On the other hand the treatment with cytoD does affect the fluorescence cytoplasma distribution indicating a major role of the cytoskeleton. The observed effect of cytoD on myosin (Fig.5C,C') seems to imply an indirect effect of cytoD on the fluorescence distribution mediated by myosin related to its role as a component of the cellular stress fibers.[15] A direct effect of myosin acting as a molecular motor seems to be excluded due to the very limited effect of metabolic poisons on the fluorescence distribution (Fig.4D).

It appears therefore reasonable to develop a physical model for the perinuclear accumulation based on the role of the cytoskeleton, whose integrity is crucial to favour the fluorophore accumulation close to the nuclear membrane, thus against a diffusion gradient. With our choice of normalized distance, $\mathrm{x}$, along the EL line, the solution of the diffusion equation for the stationary case can be written as (see Supplementary Material, "Derivation of Eq.3"):

$$
c_{e q}(x)=c_{N} \exp \left[\frac{\langle\Delta U\rangle}{K_{B} T} \frac{(x-100)}{100}\right]
$$

The comparison of Eq.1 to Eq.3 indicates that the best fit decay length, L, gives the strength of the average interaction energy gain, $\langle\Delta \mathrm{U}\rangle=\left\langle\mathrm{U}_{\text {cell }}\right\rangle-\left\langle\mathrm{U}_{\text {nucleus }}\right\rangle$, that the fluorophore suffers when moving from the cell membrane to the nuclear membrane $(\langle\Delta U\rangle \geq 0)$ :

$$
\langle\Delta U\rangle=100 \frac{K_{B} T}{L}
$$

The interaction energy gain $\Delta \mathrm{U}$ is equivalent to the action of an effective force field acting on the fluorophore over the thickness of the cytoplasm. This force field may be related to a difference in pressure between cytoplasm areas close to the cell and to the nuclear membrane. However, since from the data 
analysis (see Eqs.3, 4) we have a direct access only to the overall interaction energy gain that corresponds to this force field, we have preferred to evaluate the degree of perinuclear accumulation (i.e. the exponent in Eq. 3) in terms of the energy gain $\Delta U$.

The $\mathrm{L}$ values reported in Table $\mathbf{I}$ correspond to a decrease of $0.9 \pm 0.14 K_{B} T$ units of $\langle\Delta U\rangle$, when decreasing the temperature from $\mathrm{T}=37^{\circ} \mathrm{C}$ to $\mathrm{T}=4^{\circ} \mathrm{C}$. This may reflect a change in the cytoskeleton structure and/or dynamics that affects the fluorophore distribution.

By analyzing Eq.1 we gain also information on the relative perinuclear concentration of loaded dye at the equilibrium. In fact, if the perinuclear and the cell concentrations of the fluorophore are $c_{N}$ and $c_{C}$, respectively, the excess perinuclear concentration, $\Delta \mathrm{C}=\left(\mathrm{c}_{\mathrm{N}}-\mathrm{c}_{\mathrm{C}}\right) / \mathrm{c}_{\mathrm{C}}$, can be related to the area, $\mathrm{A}$, under the fluorescence distribution curves reported in Fig.3 (normalized to the background level $\mathrm{F}_{0}$ ) and the decay length, as (see Supplementary Material, section “Derivation of Eq.5”):

$$
\Delta_{C}=\frac{\left(c_{N}-c_{C}\right)}{c_{C}}=(A+1) \frac{100}{L}
$$

The value of $\Delta_{C}$ estimated on the plots of the fluorescence distribution (see Table I), indicates that the perinuclear excess of concentration increases by approximately 55\% when increasing the temperature from $\mathrm{T}=4{ }^{\circ} \mathrm{C}$ to $\mathrm{T}=37^{\circ} \mathrm{C}$.

The limited changes in the fluorophore internalization observed when decreasing the cell temperature, suggest that endocytotic pathways, that are sensibly dependent on the temperature [16,17] in the range $4-37^{\circ} \mathrm{C}$, are only partially effective in the upload process of the c-SLNs and that the internalization is also determined by a direct interaction between the membrane and the c-SLNs phospholipids. This picture agrees with previous analyses [10] that showed that the internalization of the c-SLNs carried fluorophore occurs with the modification of the lipid (SLNs) physical and chemical thermodynamic parameters, probably due to their interaction with phospho-lipids (membrane). Indeed, calorimetric studies indicate [10] that the cSLNs interacting with the cell membrane, only partially preserve their native structure. A fraction of the intracellular fluorescence could then result from coumarin-6 released from the SLNs upon their interaction with the cell membrane. 
The treatment with CytoD markedly reduced the inhomogeneity of the intracellular distribution (Fig.5 $\mathbf{A}^{\prime}$ ): L was as large as $80 \%$, almost three times larger than the value measured in the absence of CytoD. This finding corresponds to a decreased energy gain (see Eq.4, $\langle\Delta U\rangle \cong K_{B} T$ compared to $\langle\Delta U\rangle \cong 3$ $\mathrm{K}_{\mathrm{B}} \mathrm{T}$ prior to the CytoD treatment, Table I). It must be noted that the analysis proposed here, is not substantially affected by the assumption of a one dimensional diffusion process (see "The distribution of particles in a polar coordinate system" in the Supplementary Materials).

\section{Upload kinetics.}

Concerning the origin of the hypothesized NPs-cytoskeleton interaction, we suggest the possibility of local overpressures generated by the cytoskeleton dynamics. The cytoplasm of mammalian cells possesses actin and myosin structures arranged as stress fibers and organized to generate contractile structures responsible for cellular tension $[15,18,19]$. These fibers in intact living cells, may shorten to generate tension as well as elongate, thereby determining also cellular elasticity. One possible hypothesis about the generation of the fluorescence perinuclear accumulation in the cells may rely on the presence of these forces [8] that should be relaxed when treating the cells with cytoskeleton destabilising drugs, such as CytoD, as indeed is found here (Fig.5A, A').

We develop and discuss a simple model based on the diffusion of the fluorophore in the cytoplasm under the action of a mean field interaction force. If we assume the following initial condition, $c(x, 0)=c_{e x t} \delta(x)$, that describes the case in which the extracellular fluorophore concentration is constant, $c_{\text {ext }}$ at time $\mathrm{t}=0$, the intracellular fluorophore concentration follows a simple exponential law (Eq.B8 in the Supplementary Material):

$$
\left.c(x, t)\right|_{x=0,100}=a(x)+b(x) \exp \left[-t \Gamma_{0}\right]
$$

where $\mathrm{a}(\mathrm{x})$ and $\mathrm{b}(\mathrm{x})$ are parameters that depend on the initial fluorophore concentration at the membrane, $\mathrm{c}_{\mathrm{ext}}$, and on the position along the EL line. The relaxation rate $\Gamma_{0}$ is related to the effective energy gain, $\langle\Delta U\rangle$, the fluorophore diffusion coefficient, $\mathrm{D}$, and the average cell thickness, $\Delta$ (from the cell to the nuclear membrane) as: 


$$
\Gamma_{0}=\frac{D}{4 \Delta^{2}}\left(\frac{\Delta U}{K_{B} T}\right)^{2}
$$

A more realistic model takes into account that the SLNs enter the cell membrane at a characteristic rate, $\gamma$, which depends on the details of the nanoparticle interaction with the membrane. Moreover, the local extracellular SLNs concentration, $c_{\text {ext }}$, might decay slowly (with a second relaxation rate $\Gamma$ ) with time due to the continuous internalization of the SLNs by all the cells in the culture. Within these two hypotheses the initial conditions for the intracellular fluorophore concentration close to the cell membrane, is actually described by: $c(0, t)=c_{e x t}(1-\exp [-t \gamma]) \exp [-t \Gamma]$. The first exponential growth (the term within the round parentheses) accounts for the increase in the concentration of the fluorophore close to the cell membrane, due to the internalization process. The second exponential decay, with relaxation rate $\Gamma$, accounts instead for the local depletion of the SLNs in the cell culture again at the cell membrane. Within such model, Eq.6 changes to a sum of exponential decays (see Supplementary Material) that, with the assumption that $\Gamma \ll<$, can be written as:

$$
c(x, t) \cong B\left(\exp [-t \gamma]-\exp \left[-t \Gamma_{0}\right]\right)+c_{\infty}(x)(1-\exp [-t \gamma])
$$

From Eq.8, we can obtain the fluorescence signal along the EL as a function of time: $F(x, t) \cong F_{\infty} c(x, t) / c_{\infty}(x)=b\left(\exp [-t \gamma]-\exp \left[-t \Gamma_{0}\right]\right)+F_{\infty}(1-\exp [-t \gamma])$. We have globally fitted the two data sets in Fig.2B to Eq.8 by assuming common values of $\Gamma_{0}$ and $\gamma$, whose best fit values are: $\Gamma_{0}=0.1 \pm 0.02 \mathrm{~min}$ and $\gamma=0.07 \pm 0.02 \mathrm{~min}^{-1}$. The time delay observed in the rise of the fluorophore concentration (Fig.2B) is determined by the relaxation rate $\gamma$, and the slope of the fluorescence signal at larger times is related to the rate $\Gamma_{0}$. The marked sigmoidal shape of the kinetics derives then from the difference between the two relaxation rates, $\Gamma_{0}$ and $\gamma$, that account for the intracellular diffusion and the internalization of the fluorophore, respectively. 
From the diffusion relaxation rate, $\Gamma_{0}$, we can estimate a value of the diffusion coefficient of the fluorophore in the cytoplasm. By taking an average value of the energy loss, $\Delta \mathrm{U} / \mathrm{K}_{\mathrm{B}} \mathrm{T} \cong 2.8$ (Table 1 , without CytoD) and by assuming an average size of $5 \mu \mathrm{m}$ for the cell cytoplasm $(\Delta \cong 5 \mu \mathrm{m})$, we estimate $\mathrm{D} \cong 0.02$ $\mu \mathrm{m}^{2} / \mathrm{s}$. This value is more than four orders of magnitude lower than the coumarin- 6 diffusion in aqueous solution at room temperature $\left(\mathrm{D}_{\text {coum }} \cong 280 \mu \mathrm{m}^{2} / \mathrm{s}\right)$, and about two orders of magnitude lower than the diffusion coefficient of the SLNs in the same conditions. Literature data on the diffusion coefficient of simple fluorophore in the cell cytoplasm and on the cell membrane[20] indicate that the fluorophore diffusion decreases by almost two orders of magnitudes in the cytoplasm and about four orders of magnitude on the cell membrane. In the view of these data, our estimate of the diffusion coefficient associated with the coumarin-6 fluorescence signal suggests that the fluorophore moves within the cell cytoplasm in a form that is still reminiscent of the nanocarrier structure and not in the form of free dye. This hypothesis is also supported by the results of calorimetric studies [10] from which it seems that the c-SLNs preserve partially their native structure after entering the cell.

In conclusion, the analysis of the fluorescence microscopy data indicates that the combined action of the diffusion of the dye in the cytoplasm and of a mean force field acting on the dye induces its accumulation around the nucleus. Data collected in conditions in which the cytoskeleton is perturbed suggest that these forces, that correspond to an energy gain of the order of few $\mathrm{K}_{\mathrm{B}} \mathrm{T}$ from the cell to the nuclear membrane, originate from the cytoskeleton structure and dynamics but are not directly related to the action of molecular motors. The biophysical model that we propose and discuss analytically in the Supplementary Materials, allows us to quantify the interaction energy gain of the fluorophore from the cell to the nucleus membrane. This study also sets a warning about non-specific effects in the intracellular distribution of drugs due to the cytoskeleton structure and state. 


\section{Acknowledgements}

The research was partially funded by the European Community Contract STREP ${ }^{\circ}$ LSHB-CT2006-037639-BONSAI (Bio-imaging with Smart Functional Nanoparticles) to G.M. and by the European Community contract No: 201842, ENCITE (European Network for Cell Imaging and Tracking Expertise) to G.C. The authors thank NANOVECTOR for having kindly provided SLNs. 


\section{References}

[1] K. Sugano, M. Kansy, P. Artursson, et al. Coexistence of passive and carrier-mediated processes in drug transport. Nat Rev Drug Discov. 9 (2010) 597-614.

[2] S.K. Lai, K. Hida, C. Chen et al. Characterization of the intracellular dynamics of a non-degradative pathway accessed by polymer nanoparticles. J. Control Release. 125 (2008) 107-11.

[3] J. Suh , D. Wirtz , J. Hanes Efficient active transport of gene nanocarriers to the cell nucleus. Proc Natl Acad Sci U S A. 100 (2003) 3878-3882.

[4] M.D. Chavanpatil, A. Khdair, B. Gerard et al. Surfactant-polymer nanoparticles overcome Pglycoprotein-mediated drug efflux. Mol. Pharm. 4 (2007) 730-8.

[5] Chakraborty S.K., J.A. Fitzpatrick, J.A. Philippi, et al. Cholera toxin B conjugated quantum dots for live cell labeling. Nano Lett. 7 (2007) 2618-26

[6] S.K. Lai, K. Hida, S.T. Man, et al. Privileged delivery of polymer nanoparticles to the perinuclear region of live cells via non-clathrin, non-degradative pathway. Biomaterial, 28 (2007) 2876-2884.

[7] R.A. Gemeinhart, D. Luo, W.M. Saltzman. Cellular fate of a modular DNA delivery system mediated by silica nanoparticles. Biotechnol Prog. 21 (2005) 532-7.

[8] C. Schulze, K. Mueller, J.A. Kaes et al. Compaction of Cell Shape Occurs Before Decrease of Elasticity in CHO-K1 Cells Treated With Actin Cytoskeleton Disrupting Drug Cytochalasin D. Cell motility and the Cytoskeleton. 66 (2009) 193-201.

[9] W. Wei, G.H. Ma , G. Hu , et al. Preparation of Hierarchical Hollow CaCO3 Particles and the Application as Anticancer Drug Carrier. J. Am. Chem. Soc. 130 (2008) 15808-10.

[10] I. Rivolta, A. Panariti, B. Lettiero et al. Cellular uptake of coumarin-6 as a model drug loaded in Solid Lipid Nanoparticles (SLN). J. Physiol. Pharm. 62 (2011) In press.

[11] L. Botto, E. Beretta, A. Bulbarelli, et al. Hypoxia-induced modifications in plasma membranes and lipid microdomains in A549 cells and primary human alveolar cells. J. Cell Biochem. 105 (2008) 50313. 
[12] Tsuruta D, Gonzales M, Hopkinson SB, Otey C, Khuon S, Goldman RD, Jones JCR. Microfilament-dependent movement of the $\beta 3$ integrin subunit within focal contacts of endothelial cells. The FASEB Journal express article. Published online April 10, 2002

[13] Smith D.A. and Simmons R.M. Model of motor assisted transport of intracellular particles. Bipohysical Journal. 80 (2001) 45-68

[14] Tabb, J. S., B. J. Molyneaux, D. L. Cohen, S. A. Kuznetsov, and G. M. Langford. Transport of ER vesicles on actin filaments in neurons by myosin V. J. Cell Sci. 111(1998) 3221-3234.

[15] Y. Luo, X. Xu, T. Lele, et al. A multi-modular tensegrity model of an actin stress fiber. Biomech. 41 (2008) 2379-2387.

[16] P.H. Weigel and J.A. Oka. Temperature dependence of endocytosis mediated by the asialoglycoprotein receptor in isolated rat hepatocytes. Evidence for two potentially rate-limiting steps. J. Biol. Chem. 256 (1981) 2615-2617.

[17] W.F. Wolkers , S.A. Looper, R.A. Fontanilla, et al. Temperature dependence of fluid phase endocytosis coincides with membrane properties of pig platelets. Biochim Biophys Acta. 1612 (2003) 154-63.

[18] Goeckeler ZM, Bridgman PC, Wysolmerski RB. Nonmuscle myosin II is responsible for maintaining endothelial cell basal tone and stress fiber integrity. Am J Physiol Cell Physiol 295 (2008) C994-C1006.

[19] Y. Cai, M.P. Sheetz. Force propagation across cells: mechanical coherence of dynamic cytoskeletons. Curr. Opin. Cell Biol. 21 (2009) 47-50.

[20] P. Schwille, U. Haupts, S. Maiti, et al. Molecular dynamics in living cells observed by fluorescence correlation spectroscopy with one- and two-photon excitation. Biophys. J. 77 (1999) 2251-2265. 


\section{Figure Legends}

Figure 1. Image: an Emission Line (EL) is drawn on a representative cell image acquired after 30 min of cSLNs incubation ( $\lambda_{\text {exc }}=488 \mathrm{~nm}$, integration time $400 \mathrm{~ms}$ ). The EL runs from the cell to the perinuclear region and four different ROIs (at $0,1,2.4,3.6 \mu \mathrm{m}$ along the EL) on which the fluorescence analysis was carried out, are marked on the image. Plot: we report the representative traces of the fluorescent signal collected on a single cell along the EL and recorded at increasing incubation time from bottom to top ( $0,9,15$ and 30 min). The signal is shown in arbitrary units.

Figure 2. Panel A: fluorescence intensity as a function of the distance (estimated in normalized units $\left.x=100\left(r-r_{N}\right) /\left(r_{C}-r_{N}\right)\right)$ from the plasma membrane along the EL for $15 \mathrm{~min}$ (open squares) and $30 \mathrm{~min}$ (open circles) of cell incubation with c-SLNs. The solid lines are the best fit of Eq.1 to the data. The inset shows the normalized fluorescence signal together with the best fit of Eq.1 to both data sets. Statistical analysis was carried out by $\mathrm{t}$-test and the significance level was set at $\mathrm{p}<0.001$ (indicated by symbol $*$ in the plot). Panel B: the plot represents the fluorescence increase measured in cells incubated with c-SLNs at 50\% (open circles) and $75 \%$ (closed circles) along the EL. The data in both panels are averaged over seven cells and the standard deviations are reported. The dashed lines represent the individual fits of the EL=50\% and $\mathrm{EL}=75 \%$ data to the sigmoidal function, Eq.2, with best fit parameters: $f_{0}(50 \%)=-2.7 \pm 0.5, f_{1}(50 \%)$ $=58 \pm 10, t_{1 / 2}(50 \%)=17.5 \min \pm 4 \min$ and $\delta(50 \%)=3.5 \min \pm 1.5 \mathrm{~min}, f_{0}(75 \%)=-26 \pm 5, f_{1}(75 \%)=150 \pm 25$, $t_{1 / 2}(75 \%)=19 \min \pm 4 \min$ and $\delta(75 \%)=7 \min \pm 3 \mathrm{~min}$. The solid lines are obtained by global fitting both data sets to Eq.8. The best fit parameters are: $F_{\infty}(50 \%)=76 \pm 10, b(50 \%)=290 \pm 50, F_{\infty}(75 \%)=174 \pm 5, b(75 \%)$ $=630 \pm 55, \gamma=0.07 \pm 0.03 \mathrm{~min}^{-1}$ and $\Gamma_{0}=0.01 \pm 0.02 \mathrm{~min}^{-1}$.

Figure 3. Main panel: effect of the c-SLN incubation temperature decrease on the intracellular fluorescence: $\mathrm{T}=37^{\circ} \mathrm{C}$ (closed circles) and $\mathrm{T}=4^{\circ} \mathrm{C}$ (open circles). The data result from the average over seven cells and the error bars represent the standard deviations. The solid lines are the best fit of Eq. 1 to the data. The inset reports the residuals of the data to the best fit lines (solid line for $\mathrm{T}=4^{\circ} \mathrm{C}$ and dashed line for $\mathrm{T}=37^{\circ} \mathrm{C}$ ). 
Figure 4. Panel A: intracellular fluorescence distribution after exposure of $\mathrm{A} 30$ to c-SLN for $45 \mathrm{~min}$ at $37^{\circ} \mathrm{C}$ in control condition. Panel B and C: Intracellular fluorescence distribution after treatment with the metabolic poisons $\mathrm{NaN}_{3}$ and DDG, respectively. Panel D: quantification of the fluorescence intensity as a function of the distance from the plasma membrane along the EL in the three conditions presented above: black squares (control cell line), red circles $\left(\mathrm{NaN}_{3}\right.$ treated cell line) and green triangles (DDG treated cell line). The solid lines (same color code as the symbols) are the best fit to Eq. 1.

Figure 5. Typical images acquired on A30 cells in control condition (CTRL) and after treatment with CytoD $2 \mu \mathrm{M}$ for $1 \mathrm{~h}$ (+CytoD). Fluorescence distribution in A30 cells after $30 \mathrm{~min}$ of incubation with c-SLNs before (A) and after CytoD treatment, (A'). The treatment with CytoD breaks the major part of actin (B vs B') and myosin (C vs C') filaments in the cells, while microtubules integrity was preserved (D vs D'). The horizontal white bar corresponds to $10 \mu \mathrm{m}$. Actin and myosin filaments are labelled in red, microtubules are labelled in green, the cell nuclei in blue. Panel E: the plot represents the trend of the fluorescence as a function of the distance from the plasma membrane for the control (closed circles), the samples treated with CytoD after incubation with c-SLN (open circles), the samples treated with CytoD before incubation with cSLN (open squares). All data were normalized to the fluorescence signal measured at $E L=25 \%$. The data result from the average over ten cells and the error bars represent the standard deviations. Statistical analysis was carried out by t-test and the significance level relative to control was set at $\mathrm{p}<0.001$ (indicated by symbol \#* in the plot). 


\title{
Online supplementary material:
}

\section{Derivation of the Intracellular Equilibrium Load Distribution, Eq. 3.}

We derive the equilibrium distribution of the load assuming a Cartesian coordinate system and a constant average force field between the cell and the nuclear membrane.

\section{Derivation of the Total Intracellular Load, Eq. 5.}

We derive a possible measurement of the total intracellular load from the computation of the integral of the fluorescence signal along the emission line.

\section{Time Evolution of the Fluorescence During the Uptake: Eq.8.}

We derive a simplified expression of the time dependence of the intracellular load concentration observed during the cellular uptake.

\section{The Distribution of Particles in a Polar Coordinate System.}

We discuss the differences of the equilibrium Intracellular distribution of the load when assuming a polar and a Cartesian coordinate system.

\section{Footnote}

\author{
Abbreviations list \\ Arbitrary units (a.u.) \\ Cytochalasin D (cyto D) \\ (coumarin-6) loaded in the SLNs (c-SLNs) \\ Emission Line (EL) \\ Minutes (min) \\ Nanoparticles (NPs) \\ Solid Lipid Nanoparticles (SLNs) \\ Regions of Interest (ROIs)
}




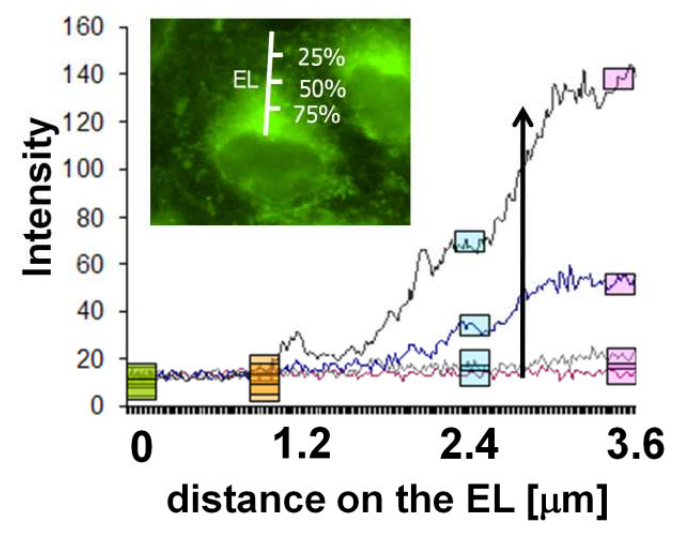

Fig. 1 


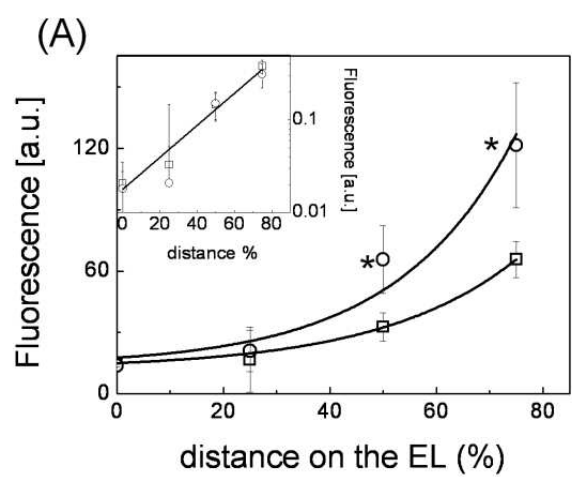

(B)

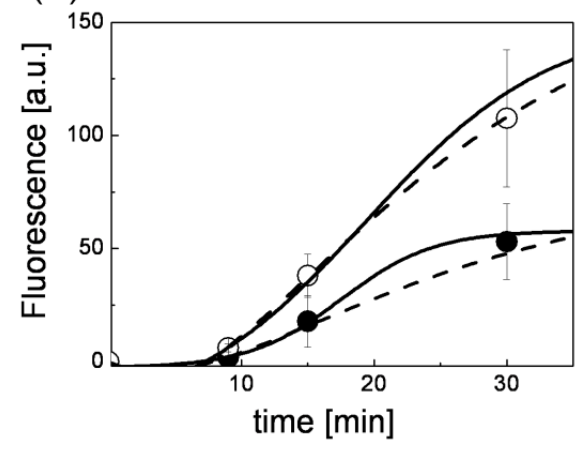

Fig. 2 


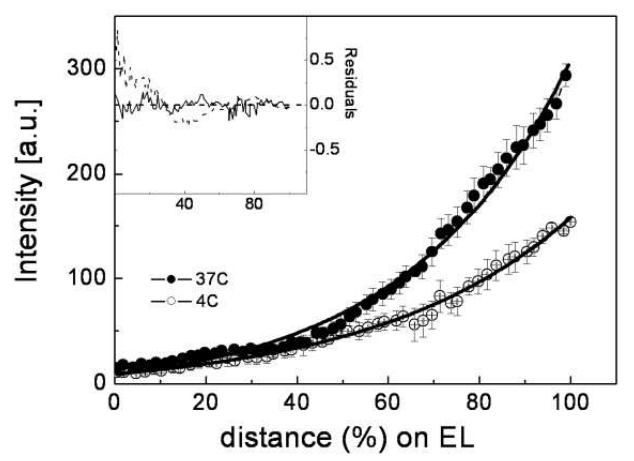

Fig. 3 

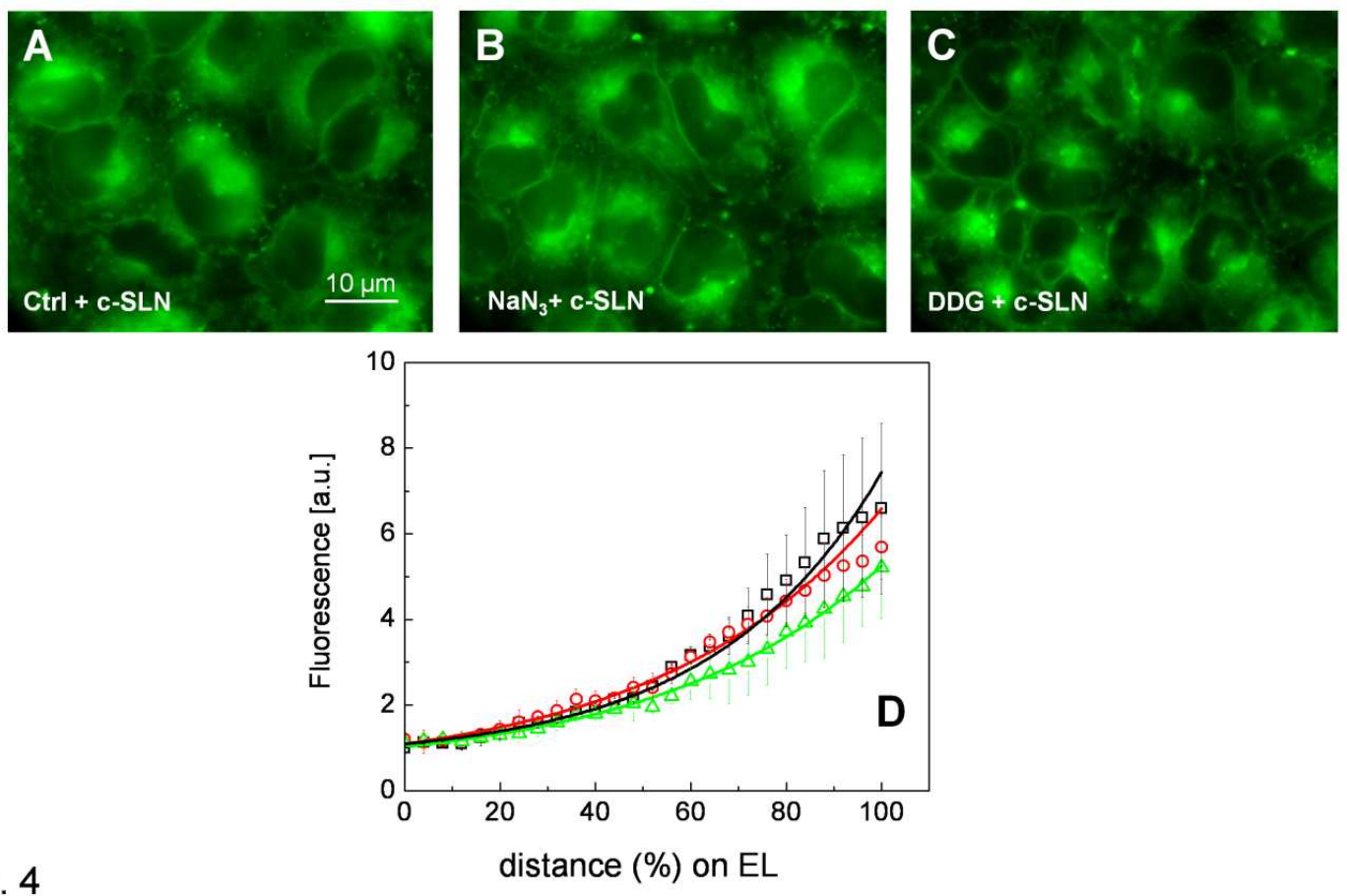

Fig. 4 

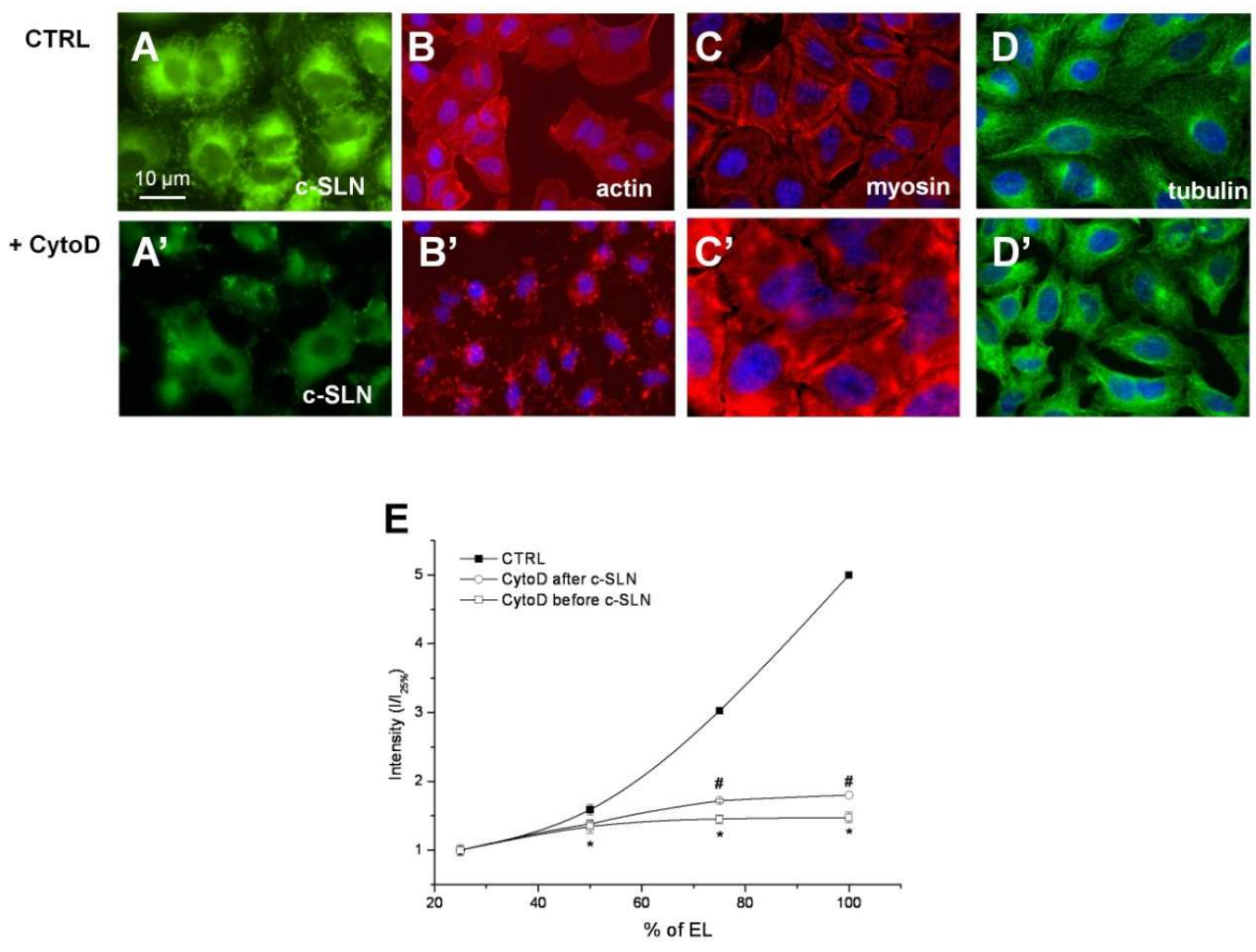

Fig. 5 


\section{Research highlights}

We have measured the cytoplasmic distribution of the coumarin-6 carried by Solid Lipid Nanoparticles in human alveolar cells and observed a perinuclear accumulation of the fluorescence that we model with the a mean field interaction of the load with the cytoskeleton. Experiments as a function of temperature, the cytoskeleton state and metabolic poisons validate this analysis. 


\section{Graphıcal Abstract}

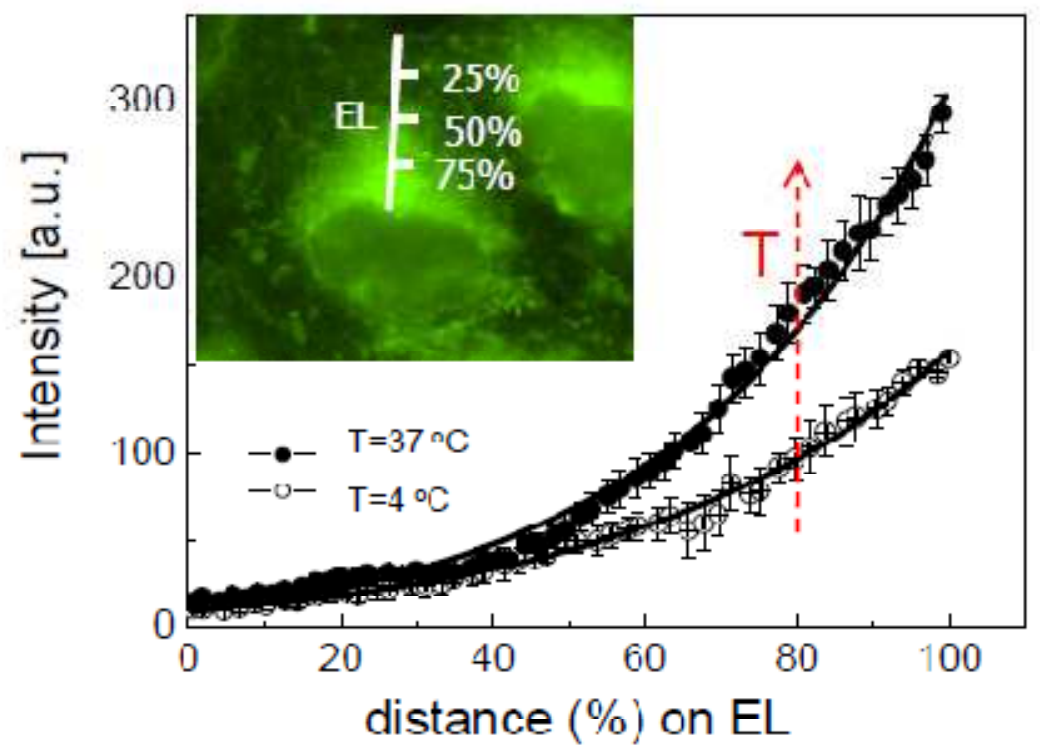


Table I. Analysis of the distribution of coumarin-6 as a function of the nuclear to cytoplasmic membranes.

\begin{tabular}{|l|l|l|l|l|l|l|}
\hline & $\mathrm{A}$ & $\mathrm{L}(\%)$ & $\mathrm{F}_{0}$ & $\mathrm{~A} / \mathrm{F}_{0}$ & $\frac{\langle\Delta U\rangle}{K_{B} T}=\frac{100}{L}$ & $\Delta_{\mathrm{C}}$ \\
\hline $\mathrm{T}=37^{\circ} \mathrm{C}$ & $315 \pm 7$ & $31 \pm 1$ & $4 \pm 2$ & $75 \pm 6$ & $3.2 \pm 0$. & $21.7 \pm 3$ \\
\hline $\mathrm{T}=4^{\circ} \mathrm{C}$ & $160+0.9$ & $43 \pm 2$ & $2 \pm 2$ & $80 \pm 8$ & $2.3 \pm 0.1$ & $13.8 \pm 2$ \\
\hline $\mathrm{T}=37^{\circ} \mathrm{C}$, with cytoD & $86 \pm 16$ & $80 \pm 40$ & $40 \pm 7$ & $2.1 \pm 1$ & $1.2 \pm 0.6$ & \\
\hline
\end{tabular}

Table 1. L is in unit of \% of the total nuclear to the cell membrane distance; the load concentration at the cell membrane is $c(x=0)=A \exp [-100 / L]$; the parameter $\Delta_{\mathrm{C}}=\left(\mathrm{c}_{\mathbb{N}}-\mathrm{c}_{\mathrm{C}}\right) / \mathrm{c}_{\mathrm{C}}$ indicates the excess of the load concentration at the nuclear membrane (see Eq.5) computed by numerical integration (trapezoidal nule) of the data in Fig.4. 